\title{
Conformal deformations of integral pinched 3-manifolds
}

\author{
Giovanni CATINO ${ }^{a}$ and Zindine DJADLI ${ }^{b}$ \\ ${ }^{a}$ SISSA \\ Via Beirut 2-4 \\ 34014 Trieste - Italy \\ ${ }^{b}$ Institut Fourier - Université Grenoble 1 \\ 100 rue des Maths \\ F38402 Saint-Martin d'Hères Cedex - France
}

ABSTRACT. In this paper we prove that, under an explicit integral pinching assumption between the $L^{2}$ norm of the Ricci curvature and the $L^{2}$-norm of the scalar curvature, a closed 3-manifold with positive scalar curvature admits a conformal metric of positive Ricci curvature. In particular, using a result of Hamilton, this implies that the manifold is diffeomorphic to a quotient of $\mathbb{S}^{3}$. The proof of the main result of the paper is based on ideas developped in an article by M. Gursky and J. Viaclovsky.

Key Words: geometry of 3-manifolds, rigidity, conformal geometry, fully non-linear equation

\section{AMS subject classification: $53 \mathrm{C} 24,53 \mathrm{C} 20,53 \mathrm{C} 21,53 \mathrm{C} 25$}

\section{Introduction}

Ever since Poincaré asked his now famous question about the 3-sphere, mathematicians have been absorbed by the problem of trying to capture the topological or geometrical properties of a manifold by its metric structure. One of the basic questions is: under which conditions on the curvature tensor a Riemannian manifold is homeomorphic (or diffeomorphic) to a space form (a manifold of constant sectional curvature)?

In 1951 H. E. Rauch introduced in [15] the idea of curvature pinching for Riemannian manifolds and posed the question whether a simply connected compact manifold $M^{n}$ whose sectional curvatures all lie in the interval $(1,4]$ is necessarily homeomorphic (or diffeomorphic) to the standard sphere $\mathbb{S}^{n}$. A result of this type is usually referred to as a Sphere Theorem. After a lot of research in this field, we know that the answer is positive, due to the works of Berger and Klingenberg for the topological statement and Brendle-Schoen [2] for the more general result on the differentiable sphere theorem, namely:

Theorem 1.1 (The Sphere Theorem) (Brendle-Schoen [2]) Let $(M, g)$ be a compact Riemannian manifold with 1/4-pinched curvature (that is $(M, g)$ has positive sectional curvature and the ratio of the minimum and the maximum of the sectional curvatures is always strictly bigger than a quarter). Then $M$ admits a metric of constant sectional curvature, therefore is diffeomorphic to a spherical space form.

In two dimension, due to Koebe and Poincaré, the Uniformization Theorem for surfaces asserts that any compact (orientable) surface $M$, admits a Riemannian metric $g$ of constant curvature $k_{g}=+1,0,-1$. Moreover, according to the Gauss-Bonnet formula, for every metric $g$, one has

$$
\int_{M} k_{g} d V_{g}=2 \pi \chi(M)
$$

hence, any surface belongs to a unique geometric type (spherical, Euclidean or hyperbolic) determined by the topology (namely the Euler-Poincaré characteristic $\chi(M)$ ). In other words in two dimensions an

\footnotetext{
${ }^{1}$ E-mail addresses: catino@sissa.it, Zindine.Djadli@ujf-grenoble.fr
} 
integral pinching condition (as the positivity of the above integral) implies the geometrical conclusion, encoded in the Euler-Poincaré characteristic, that the manifold is diffeomorphic to a spherical space form.

In the present work we will concentrate on similar kind of results in dimension higher than two, with particular attention to the following question: is it possible, like in the case of surfaces, to characterize spherical space forms by looking at an integral pinching condition instead of a pointwise one?

The first attempt in generalizing a pointwise to an integral pinching condition was done by S.-Y. A. Chang, M. Gursky and P. C. Yang in [4]. They showed that the sphere in dimension four can be characterized by an integral curvature condition. Later on M. Gursky and J. Viaclovsky [12] gave a simpler proof of this theorem

Theorem 1.2 (Chang-Gursky-Yang [4] - Gursky-Viaclovsky [12]) Let $\left(M^{4}, g\right)$ be a closed Riemannian 4-manifold with positive scalar curvature. Assume that

$$
\int_{M}\left|R c_{g}\right|^{2} d V_{g}<\frac{1}{3} \int_{M} R_{g}^{2} d V_{g}
$$

Then $M$ admits a metric $\tilde{g}$ conformal to $g$ such that $R c_{\tilde{g}}>0$.

In this paper we extend to the case of three manifolds the technique used in [12] in order to prove this Theorem. Our proof is heavily based on the proof given by M. Gursky and J. Viaclovsky [12] of the theorem stated above.

Before stating our main result it will be useful to recall some well-known facts. In 1982, Hamilton [13] introduced the Ricci flow in order to study "dynamically" the relationships between topology and curvature of manifolds. He proved that the metric of any 3-dimensional compact manifolds with positive Ricci curvature can be deformed, via the Ricci flow, into a metric of constant positive sectional curvature (and it follows that the manifold is diffeomorphic to a spherical space form).

In dimension three and in presence of positive scalar curvature, the positivity of the Ricci tensor is implied by a pointwise pinching condition as $\left|R c_{g}\right|_{g}^{2} \leq \frac{3}{8} R_{g}^{2}$. What we prove here is that this pinching condition can be replaced by an integral one. Here is our main result:

Theorem 1.3 Let $(M, g)$ be a closed 3-dimensional Riemannian manifold with positive scalar curvature. If

$$
\int_{M}\left|R c_{g}\right|_{g}^{2} d V_{g} \leq \frac{3}{8} \int_{M} R_{g}^{2} d V_{g}
$$

then there exists a metric $\tilde{g}$ conformal to $g$ such that $R c_{\tilde{g}}$ is everywhere positive. In particular, using a result of Hamilton [13], it follows that $M$ is diffeomorphic to a spherical space form.

Let us emphasize the fact that, in our results, we don't make any assumption on the positivity of the Ricci tensor, we only assume that its trace is positive and a pinching on its $L^{2}$-norm.

During the preparation of the manuscript of this paper, we learned that Y. Ge, C.S. Lin and G. Wang [6] proved a weaker version of our Theorem, namely they prove that if $(M, g)$ is a closed 3-dimensional Riemannian manifold with positive scalar curvature and if $\int_{M}\left|R c_{g}\right|_{g}^{2} d V_{g}<\frac{3}{8} \int_{M} R_{g}^{2} d V_{g}$ then $M$ is diffeomorphic to a spherical space form. Their proof is completely different from ours since they use a very specific conformal flow.

There is a way to relate these result to the so-called $Q$-curvature (the curvature associated to the Paneitz operator). The Paneitz operator introduced by Paneitz in [14] has demonstrated its importance in dimension 4 (see for example Chang-Gursky-Yang [3] and [4]). In dimension 3, the $Q$-curvature is defined by

$$
Q_{g}=-\frac{1}{4} \Delta_{g} R_{g}-2\left|R i c_{g}\right|_{g}^{2}+\frac{23}{32} R_{g}^{2}
$$

the Paneitz operator being defined (in dimension 3) by

$$
P_{g}=\Delta_{g}^{2}-\operatorname{div}_{g}\left(-\frac{5}{4} R_{g} g+4 R i c_{g}\right) d-\frac{1}{2} Q_{g}
$$

The Paneitz operator satisfies the conformal covariant property, that is, if $\rho \in C^{\infty}(M), \rho>0$, then for all $\varphi \in C^{\infty}(M), P_{\rho^{-4} g}(\varphi)=\rho^{7} P_{g}(\rho \varphi)$. We can now state the Corollary (here $[g]$ stands for the conformal class of $g)$ : 
Corollary 1.4 Let $(M, g)$ be a closed 3-dimensional Riemannian manifold with non-negative Yamabe invariant. If there exists a metric $g^{\prime} \in[g]$ such that the $Q$-curvature of $g^{\prime}$ satisfies

$$
Q_{g^{\prime}} \geq \frac{1}{48} R_{g^{\prime}}^{2}
$$

then $M$ is diffeomorphic to a quotient of $\mathbb{R}^{3}$ if $Y(M,[g])=0$ or to a spherical space form if $Y(M,[g])>0$.

Aknowledgements : The authors would like to thank Sun-Yung Alice Chang, Matt Gursky and Paul Yang for their interest in this work.

\section{Ellipticity, upper bound and gradient estimate}

Consider $(M, g)$, a compact, smooth, 3-dimensional Riemannian manifold without boundary. Given a section $A$ of the bundle of symmetric two tensors, we can use the metric to raise an index and view $A$ as a tensor of type $(1,1)$, or equivalently as a section of $\operatorname{End}(T M)$. This allows us to define $\sigma_{2}\left(g^{-1} A\right)$ the second elementary function of the eigenvalues of $g^{-1} A$, namely, if we denote by $\lambda_{1}, \lambda_{2}$ and $\lambda_{3}$ these eigenvalues

$$
\sigma_{2}\left(g^{-1} A\right)=\lambda_{1} \lambda_{2}+\lambda_{1} \lambda_{3}+\lambda_{2} \lambda_{3} .
$$

In this paper we choose the tensor (here $t$ is a real number)

$$
A_{g}^{t}=R i c_{g}-\frac{t}{4} R_{g} g
$$

Note that for $t=1, A_{g}^{1}$ is the classical Schouten tensor $A_{g}^{1}=R i c_{g}-\frac{1}{4} R_{g} g$ (see [1]). Hence, with our notations, $\sigma_{2}\left(g^{-1} A_{g}^{t}\right)$ denotes the second elementary symmetric function of the eigenvalues of $g^{-1} A_{g}^{t}$. We will denote $Y(M,[g])$ the Yamabe invariant associated to $(M, g)$ (here $[g]$ is the conformal class of the metric $g$, that is $[g]:=\left\{\tilde{g}=e^{-2 u} g\right.$ for $\left.\left.u \in C^{\infty}(M)\right\}\right)$. We recall that

$$
Y(M,[g]):=\inf _{\tilde{g} \in[g]} \frac{\int_{M} R_{\tilde{g}} d V_{\tilde{g}}}{\left(\int_{M} d V_{\tilde{g}}\right)^{\frac{1}{3}}}
$$

An important fact that will be useful is that if $g$ has positive scalar curvature then $Y(M,[g])>0$.

For the proof of our main Theorem, we will be concerned with the following equation for a conformal metric $\tilde{g}=e^{-2 u} g$ :

$$
\left(\sigma_{2}\left(g^{-1} A_{\tilde{g}}^{t}\right)\right)^{1 / 2}=f e^{2 u},
$$

where $f$ is a positive function on $M$. A simple computation, similar to that in [12], shows that this equation is equivalent to

$$
\sigma_{2}\left(g^{-1}\left(A_{g}^{t}+\nabla_{g}^{2} u+(1-t)\left(\Delta_{g} u\right) g+d u \otimes d u-\frac{2-t}{2}\left|\nabla_{g} u\right|_{g}^{2} g\right)\right)^{1 / 2}=f(x) e^{2 u}
$$

Following [12], we will discuss the ellipticity properties of equation (1).

Definition 2.1 Let $\left(\lambda_{1}, \lambda_{2}, \lambda_{3}\right) \in \mathbb{R}^{3}$. We view the second elementary symmetric function as a function on $\mathbb{R}^{3}$ :

$$
\sigma_{2}\left(\lambda_{1}, \lambda_{2}, \lambda_{3}\right)=\sum_{1 \leq i<j \leq 3} \lambda_{i} \lambda_{j}
$$

and we define

$$
\Gamma_{2}^{+}=\left\{\sigma_{2}\left(\lambda_{1}, \lambda_{2}, \lambda_{3}\right)>0\right\} \cap\left\{\sigma_{1}\left(\lambda_{1}, \lambda_{2}, \lambda_{3}\right)>0\right\} \subset \mathbb{R}^{3},
$$

where $\sigma_{1}\left(\lambda_{1}, \lambda_{2}, \lambda_{3}\right)=\lambda_{1}+\lambda_{2}+\lambda_{3}$ denotes the trace. 
For a symmetric linear transformation $A: V \rightarrow V$, where $V$ is an $n$-dimensional inner product space, the notation $A \in \Gamma_{2}^{+}$will mean that the eigenvalues of $A$ lie in the corresponding set. We note that this notation also makes sense for a symmetric 2-tensor on a Riemannian manifold. If $A \in \Gamma_{2}^{+}$, let $\sigma_{2}^{1 / 2}(A)=\left\{\sigma_{2}(A)\right\}^{1 / 2}$.

Definition 2.2 Let $A: V \rightarrow V$, where $V$ is an n-dimensional inner product space. The first Newton transformation associated with $A$ is (here $I$ is the identity map on $V$ ) $T_{1}(A):=\sigma_{1}(A) \cdot I-A$. Also, for $t \in \mathbb{R}$ we define the linear transformation $\mathcal{L}^{t}(A):=T_{1}(A)+(1-t) \sigma_{1}\left(T_{1}(A)\right) \cdot I$.

We have the following:

Lemma 2.3 If $A: \mathbb{R} \rightarrow \operatorname{Hom}(V, V)$, then $\frac{d}{d s} \sigma_{2}(A)(s)=\sum_{i, j} T_{1}(A)_{i j}(s) \frac{d}{d s}(A)_{i j}(s)$, i.e, the first Newton transformation is what arises from differentiation of $\sigma_{2}$.

Proof The proof of this lemma is a consequence of an easy computation. See Gursky-Viaclovsky [11]

Proposition 2.4 (Ellipticity property) Let $u \in C^{2}(M)$ be a solution of equation (1) for some $t \leq 2 / 3$ and let $\tilde{g}=e^{-2 u} \mathrm{~g}$. Assume that $A_{\tilde{g}}^{t} \in \Gamma_{2}^{+}$. Then the linearized operator at $u, \mathcal{L}^{t}: C^{2, \alpha}(M) \rightarrow C^{\alpha}(M)$, is invertible $(0<\alpha<1)$.

Proof The proof of this proposition, adapted in dimension 3, may be found in [12].

Throughout the sequel, $(M, g)$ will be a closed 3-dimensional Riemannian manifold with positive scalar curvature. Since $R_{g}>0$, there exists $\delta>-\infty$ such that $A_{g}^{\delta}$ is positive definite (i.e. $R i c_{g}-\frac{\delta}{4} R_{g} g>0$ on $M)$. Note that $\delta$ only depends on $(M, g)$. For $t \in[\delta, 2 / 3]$, consider the path of equations (in the sequel we use the notation $A_{u_{t}}^{t}:=A_{g_{t}}^{t}$ for $g_{t}$ given by $g_{t}=e^{-2 u_{t}} g$ )

$$
\sigma_{2}^{1 / 2}\left(g^{-1} A_{u_{t}}^{t}\right)=f e^{2 u_{t}}
$$

where $f=\sigma_{2}^{1 / 2}\left(g^{-1} A_{g}^{\delta}\right)>0$. Note that $u \equiv 0$ is a solution of (2) for $t=\delta$.

Proposition 2.5 (Upper bound) Let $u_{t} \in C^{2}(M)$ be a solution of (2) for some $t \in[\delta, 2 / 3]$. Then $u_{t} \leq \bar{\delta}$, where $\bar{\delta}$ depends only on $(M, g)$.

Proof From Newton's inequality $\sqrt{3} \sigma_{2}^{1 / 2} \leq \sigma_{1}$, so for all $x \in M, \sqrt{3} f e^{2 u_{t}} \leq \sigma_{1}\left(g^{-1} A_{u_{t}}^{t}\right)$. Let $p \in M$ be a maximum of $u_{t}$, then using the fact that

$$
A_{\tilde{g}}^{t}=A_{g}^{t}+\nabla_{g}^{2} u+(1-t)\left(\Delta_{g} u\right) g+d u \otimes d u-\frac{2-t}{2}\left|\nabla_{g} u\right|_{g}^{2} g
$$

since the gradient terms vanish at $p$ and $\left(\Delta u_{t}\right)(p) \leq 0$,

$$
\begin{aligned}
\sqrt{3} f(p) e^{2 u_{t}(p)} & \leq \sigma_{1}\left(g^{-1} A_{u_{t}}^{t}\right)(p)=\sigma_{1}\left(g^{-1} A_{g}^{t}\right)(p)+(4-3 t)\left(\Delta u_{t}\right)(p) \\
& \leq \sigma_{1}\left(g^{-1} A_{g}^{t}\right)(p)
\end{aligned}
$$

Since $t \geq \delta$, this implies $u_{t} \leq \bar{\delta}$, for some $\bar{\delta}$ depending only on $(M, g)$.

Proposition 2.6 (Gradient estimate) Let $u_{t} \in C^{3}(M)$ be a solution of (2) for some $\delta \leq t \leq 2 / 3$. Assume that $u_{t} \leq \bar{\delta}$. Then $\left\|\nabla_{g} u\right\|_{g, \infty}<C_{1}$, where $C_{1}$ depends only on $(M, g)$ and $\bar{\delta}$.

The proof of this lemma can be found in the paper Gursky-Viaclovsky [12].

Remark 2.7 Note that we will use this proposition with $\bar{\delta}$ given by Proposition 2.5 and then, since $\bar{\delta}$ depends only on $(M, g)$, we infer that $C_{1}$ only depends on $(M, g)$. 


\section{Lower bound}

For the lower bound, we need the following lemmas:

Lemma 3.1 For a conformal metric $\tilde{g}=e^{-2 u}$, we have the following integral transformation

$$
\begin{aligned}
\int_{M} \sigma_{2}\left(\tilde{g}^{-1} A_{\tilde{g}}^{1}\right) e^{-4 u} d V_{g}= & \int_{M} \sigma_{2}\left(g^{-1} A_{g}^{1}\right) d V_{g}+\frac{1}{8} \int_{M} R_{g}\left|\nabla_{g} u\right|_{g}^{2} d V_{g}-\frac{1}{4} \int_{M}\left|\nabla_{g} u\right|_{g}^{4} d V_{g} \\
& +\frac{1}{2} \int_{M} \Delta_{g} u\left|\nabla_{g} u\right|_{g}^{2} d V_{g}-\frac{1}{2} \int_{M} A_{g}^{1}\left(\nabla_{g} u, \nabla_{g} u\right) d V_{g} .
\end{aligned}
$$

Proof The proof of this relation being contained in the paper Ge-Wang [7] we omit it.

In the sequel of the proof, we will need the following proposition (see [12] for the proof)

Proposition 3.2 If for some metric $g_{1}$ on $M$ we have $A_{g_{1}}^{t} \in \Gamma_{2}^{+}$, then

$$
-A_{g_{1}}^{t}+\sigma_{1}\left(g_{1}^{-1} A_{g_{1}}^{t}\right) g_{1}>0 \quad \text { and } \quad A_{g_{1}}^{t}+\frac{1}{3} \sigma_{1}\left(g_{1}^{-1} A_{g_{1}}^{t}\right) g_{1}>0 .
$$

Going on with the proof for the lower bound, we have the Lemma:

Lemma 3.3 If $A_{\tilde{g}}^{t} \in \Gamma_{2}^{+}$, then we have the following estimate

$$
\frac{1}{2} \int_{M} A_{g}\left(\nabla_{g} u, \nabla_{g} u\right) d V_{g}<\frac{3-2 t}{8} \int_{M} R_{\tilde{g}}\left|\nabla_{g} u\right|_{g}^{2} e^{-2 u} d V_{g}+\frac{1}{4} \int_{M} \Delta_{g} u\left|\nabla_{g} u\right|_{g}^{2} d V_{g}-\frac{1}{4} \int_{M}\left|\nabla_{g} u\right|_{g}^{4} d V_{g} .
$$

Proof Since $A_{\tilde{g}}^{t} \in \Gamma_{2}^{+}$, by Proposition 3.2, we get

$$
-A_{\tilde{g}}^{t}>-\sigma_{1}\left(\tilde{g}^{-1} A_{\tilde{g}}^{t}\right) \tilde{g}=-(4-3 t) \sigma_{1}\left(\tilde{g}^{-1} A_{\tilde{g}}^{1}\right) e^{-2 u} g .
$$

Hence we get

$$
-A_{\tilde{g}}^{1}-(1-t) \sigma_{1}\left(\tilde{g}^{-1} A_{\tilde{g}}^{1}\right) e^{-2 u} g>-(4-3 t) \sigma_{1}\left(\tilde{g}^{-1} A_{\tilde{g}}^{1}\right) e^{-2 u} g,
$$

which implies that

$$
A_{\tilde{g}}^{1}<(3-2 t) \sigma_{1}\left(\tilde{g}^{-1} A_{\tilde{g}}^{1}\right) e^{-2 u} g .
$$

Applying this to $\nabla_{g} u$ we obtain

$$
\frac{1}{2} A_{\tilde{g}}^{1}\left(\nabla_{g} u, \nabla_{g} u\right)<\frac{3-2 t}{8} R_{\tilde{g}}\left|\nabla_{g} u\right|_{g}^{2} e^{-2 u} .
$$

Using the conformal transformation law of the tensor $A_{\tilde{g}}^{1}$, integrating over $M$, we have the result.

Now we are able to prove the following lower bound (recall that $C_{1}$ is given by Lemma 2.6)

Proposition 3.4 (Lower Bound) Assume that for some $t \in[\delta, 2 / 3]$ the following estimate holds

$$
\int_{M} \sigma_{2}\left(g^{-1} A_{g}^{1}\right) d V_{g}+\frac{1}{24}\left(\frac{7}{10}-t\right) \inf _{g^{\prime}=e^{-2 \varphi} g,\left|\nabla_{g} \varphi\right|_{g} \leq C_{1}}\left(\int_{M} R_{g^{\prime}}^{2} e^{-\varphi} d V_{g^{\prime}}\right):=\mu_{t}>0 .
$$

Then there exists $\underline{\delta}$ depending only on $(M, g)$ such that if $u_{t} \in C^{2}(M)$ is a solution of (2) and if $A_{u_{t}}^{t} \in \Gamma_{2}^{+}$ then $u_{t} \geq \underline{\delta}$.

Proof Since $A_{g}^{t}=A_{g}^{1}+(1-t) \sigma_{1}\left(g^{-1} A_{g}^{1}\right) g$, we easily have that

$$
\sigma_{2}\left(A_{g}^{t}\right)=\sigma_{2}\left(A_{g}^{1}\right)+(1-t)(5-3 t) \sigma_{1}\left(g^{-1} A_{g}^{1}\right)^{2} .
$$

Letting $\tilde{g}=e^{-2 u_{t}} g$,

$$
\begin{aligned}
e^{4 u_{t}} f^{2}=\sigma_{2}\left(g^{-1} A_{u_{t}}^{t}\right) & =\sigma_{2}\left(g^{-1} A_{u_{t}}^{1}\right)+(1-t)(5-3 t)\left(\sigma_{1}\left(g^{-1} A_{u_{t}}^{1}\right)\right)^{2} \\
& =e^{-4 u_{t}}\left(\sigma_{2}\left(\tilde{g}^{-1} A_{u_{t}}^{1}\right)+\frac{1}{16}(1-t)(5-3 t) R_{\tilde{g}}^{2}\right) .
\end{aligned}
$$


Integrating this with respect to $d V_{g}$, we obtain

$$
\begin{aligned}
C \int_{M} e^{4 u_{t}} d V_{g} & \geq \int_{M} f^{2} e^{4 u_{t}} d V_{g} \\
& =\int_{M} \sigma_{2}\left(\tilde{g}^{-1} A_{u_{t}}^{1}\right) e^{-4 u_{t}} d V_{g}+\frac{1}{16}(1-t)(5-3 t) \int_{M} R_{\tilde{g}}^{2} e^{-4 u_{t}} d V_{g} \\
& =\int_{M} \sigma_{2}\left(\tilde{g}^{-1} A_{u_{t}}^{1}\right) e^{-4 u_{t}} d V_{g}+\frac{1}{16}(1-t)(5-3 t) \int_{M} R_{\tilde{g}}^{2} e^{-u_{t}} d V_{\tilde{g}}
\end{aligned}
$$

where $C>0$ is chosen so that $f^{2} \leq C$ (recall that, since $f=\sigma_{2}\left(g^{-1} A_{g}^{\delta}\right), C$ depends only on $(M, g)$ ). Using the fact that

$$
R_{\tilde{g}} e^{-2 u_{t}}=R_{g}+4 \Delta_{g} u_{t}-2\left|\nabla_{g} u_{t}\right|_{g}^{2}
$$

from Lemma 3.1, we get

$$
\begin{aligned}
\int_{M} \sigma_{2}\left(\tilde{g}^{-1} A_{u_{t}}^{1}\right) e^{-4 u_{t}} d V_{g}= & \int_{M} \sigma_{2}\left(g^{-1} A_{g}^{1}\right) d V_{g}+\frac{1}{8} \int_{M} R_{\tilde{g}}\left|\nabla_{g} u_{t}\right|_{g}^{2} e^{-2 u_{t}} d V_{g} \\
& -\frac{1}{2} \int_{M} A_{g}^{1}\left(\nabla_{g} u, \nabla_{g} u\right) d V_{g} .
\end{aligned}
$$

Notice that, since $A_{u_{t}}^{t} \in \Gamma_{2}^{+}$, we have

$$
0<\sigma_{1}\left(g^{-1} A_{u_{t}}^{t}\right)=(4-3 t) \sigma_{1}\left(g^{-1} A_{u_{t}}^{1}\right),
$$

and so $R_{\tilde{g}}>0$. By Lemma 3.3, we obtain

$$
\begin{aligned}
\int_{M} \sigma_{2}\left(\tilde{g}^{-1} A_{u_{t}}^{1}\right) e^{-4 u_{t}} d V_{g} \geq & \int_{M} \sigma_{2}\left(g^{-1} A_{g}^{1}\right) d V_{g}-\frac{1-t}{4} \int_{M} R_{\tilde{g}}\left|\nabla_{g} u_{t}\right|_{g}^{2} e^{-2 u_{t}} d V_{g} \\
& -\frac{1}{4} \int_{M} \Delta_{g} u_{t}\left|\nabla_{g} u_{t}\right|_{g}^{2} d V_{g}+\frac{1}{4} \int_{M}\left|\nabla_{g} u_{t}\right|_{g}^{4} d V_{g} .
\end{aligned}
$$

By Young's inequality, one has

$$
\int_{M} R_{\tilde{g}}^{2} e^{-u_{t}} d V_{\tilde{g}} \geq \frac{2}{\varepsilon} \int_{M} R_{\tilde{g}}\left|\nabla_{g} u_{t}\right|_{g}^{2} e^{-2 u_{t}} d V_{g}-\frac{1}{\varepsilon^{2}} \int_{M}\left|\nabla_{g} u_{t}\right|_{g}^{4} d V_{g},
$$

for all $\varepsilon>0$. By an easy computation, we have

$$
\frac{1}{16}(1-t)(5-3 t)=\frac{1}{24}\left(\frac{7}{10}-t\right)+P_{2}(t),
$$

where $P_{2}(t)$ is a positive, second order, polynomial in $t$. Putting all together, we obtain (for $C>0$ depending only on $(M, g)$ )

$$
\begin{aligned}
C \int_{M} e^{4 u_{t}} d V_{g} \geq & \int_{M} \sigma_{2}\left(\tilde{g}^{-1} A_{u_{t}}^{1}\right) e^{-4 u_{t}} d V_{g}+\frac{1}{16}(1-t)(5-3 t) \int_{M} R_{\tilde{g}}^{2} e^{-u_{t}} d V_{\tilde{g}} \\
= & \int_{M} \sigma_{2}\left(\tilde{g}^{-1} A_{u_{t}}^{1}\right) e^{-4 u_{t}} d V_{g}+\left(\frac{1}{24}\left(\frac{7}{10}-t\right)+P_{2}(t)\right) \int_{M} R_{\tilde{g}}^{2} e^{-u_{t}} d V_{\tilde{g}} \\
\geq & \int_{M} \sigma_{2}\left(g^{-1} A_{g}^{1}\right) d V_{g}+\frac{1}{24}\left(\frac{7}{10}-t\right) \int_{M} R_{\tilde{g}}^{2} e^{-u_{t}} d V_{\tilde{g}} \\
& +P_{2}(t) \int_{M} R_{\tilde{g}}^{2} e^{-u_{t}} d V_{\tilde{g}}-\frac{1-t}{4} \int_{M} R_{\tilde{g}}\left|\nabla_{g} u_{t}\right|_{g}^{2} e^{-2 u_{t}} d V_{g} \\
& -\frac{1}{4} \int_{M} \Delta_{g} u_{t}\left|\nabla_{g} u_{t}\right|_{g}^{2} d V_{g}+\frac{1}{4} \int_{M}\left|\nabla_{g} u_{t}\right|_{g}^{4} d V_{g} .
\end{aligned}
$$


Now using Young's inequality and the conformal change equation of the scalar curvature, we get (for a certain $C>0$ depending only on $(M, g))$

$$
\begin{aligned}
C \int_{M} e^{4 u_{t}} d V_{g} \geq & \int_{M} \sigma_{2}\left(g^{-1} A_{g}^{1}\right) d V_{g}+\frac{1}{24}\left(\frac{7}{10}-t\right) \int_{M} R_{\tilde{g}}^{2} e^{-u_{t}} d V_{\tilde{g}} \\
& +\left(\frac{2 P_{2}(t)}{\varepsilon}-\frac{1-t}{4}\right) \int_{M} R_{g}\left|\nabla_{g} u_{t}\right|_{g}^{2} d V_{g} \\
& +\left(\frac{8 P_{2}(t)}{\varepsilon}-(1-t)-\frac{1}{4}\right) \int_{M} \Delta_{g} u_{t}\left|\nabla_{g} u_{t}\right|_{g}^{2} d V_{g} \\
& +\left(\frac{3-2 t}{4}-\frac{P_{2}(t)}{\varepsilon^{2}}-\frac{4 P_{2}(t)}{\varepsilon}\right) \int_{M}\left|\nabla_{g} u_{t}\right|_{g}^{4} d V_{g}
\end{aligned}
$$

We choose $\varepsilon=\varepsilon(t)>0$, such that $\frac{8 P_{2}(t)}{\varepsilon}-(1-t)-\frac{1}{4}=0$. One can easily check that, with this choice,

$$
\frac{2 P_{2}(t)}{\varepsilon}-\frac{1-t}{4} \geq 0 \quad \text { and } \quad \frac{3-2 t}{4}-\frac{P_{2}(t)}{\varepsilon^{2}}-\frac{4 P_{2}(t)}{\varepsilon} \geq 0 .
$$

Finally, recalling that according to lemma $2.6\left\|\nabla_{g} u_{t}\right\|_{g, \infty} \leq C_{1}$ with $C_{1}$ depending only on $(M, g)$, we obtain the following estimate (for a certain $C>0$ depending only on $(M, g)$ )

$$
\begin{aligned}
C \int_{M} e^{4 u_{t}} d V_{g} & \geq \int_{M} \sigma_{2}\left(g^{-1} A_{g}^{1}\right) d V_{g}+\frac{1}{24}\left(\frac{7}{10}-t\right) \int_{M} R_{\tilde{g}}^{2} e^{-u_{t}} d V_{\tilde{g}} \\
& \geq \int_{M} \sigma_{2}\left(g^{-1} A_{g}^{1}\right) d V_{g}+\frac{1}{24}\left(\frac{7}{10}-t\right)_{g^{\prime}=e^{-2 \varphi} g,\left|\nabla_{g} \varphi\right|_{g} \leq C_{1}}\left(\int_{M} R_{g^{\prime}}^{2} e^{-\varphi} d V_{g^{\prime}}\right) .
\end{aligned}
$$

Consider the following quantity:

$$
I(M, g):=\inf _{g^{\prime}=e^{-2 \varphi} g,\left|\nabla_{g} \varphi\right| \leq C_{1}}\left(\int_{M} R_{g^{\prime}}^{2} e^{-\varphi} d V_{g^{\prime}}\right) .
$$

We let, for $g^{\prime}=e^{-2 \varphi} g, i\left(g^{\prime}\right):=\int_{M} R_{g^{\prime}}^{2} e^{-\varphi} d V_{g^{\prime}}$. As one can easily check, if two metrics $g_{1}$ and $g_{2}$ are homothetic, then $i\left(g_{1}\right)=i\left(g_{2}\right)$. So, we have

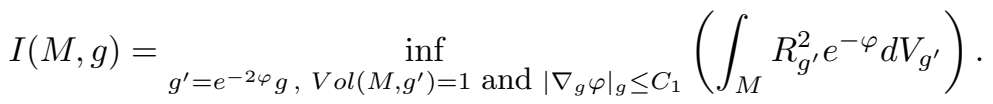

Take $\varphi \in C^{\infty}(M)$ such that, for $g^{\prime}=e^{-2 \varphi} g, \operatorname{Vol}\left(M, g^{\prime}\right)=1$ and such that $\left|\nabla_{g} \varphi\right|_{g} \leq C_{1}$ where $C_{1}$ is given by Proposition 2.6. Since $\operatorname{Vol}\left(M, g^{\prime}\right)=1$, if $p$ is a point where $\varphi$ attains its minimum we have

$$
e^{-3 \varphi(p)} \operatorname{Vol}(M, g) \geq 1,
$$

and then, there exists $C_{0}$ depending only on $(M, g)$ such that $\varphi(p) \leq C_{0}$. Now, using the mean value theorem, it follows since $\left|\nabla_{g} \varphi\right|_{g}$ is controlled by a constant depending only on $(M, g)$, that $\max \varphi \leq C_{0}^{\prime}$ where $C_{0}^{\prime}$ depends only on $(M, g)$.

Using this, we clearly have that

$$
\int_{M} R_{g^{\prime}}^{2} e^{-\varphi} d V_{g^{\prime}} \geq e^{-C_{0}^{\prime}} \int_{M} R_{g^{\prime}}^{2} d V_{g^{\prime}}
$$

Using Hölder inequality and the definition of the Yamabe invariant, we get (recall that $\operatorname{Vol}\left(M, g^{\prime}\right)=1$ )

$$
\int_{M} R_{g^{\prime}}^{2} e^{-\varphi} d V_{g^{\prime}} \geq e^{-C_{0}^{\prime}}(Y(M,[g]))^{2},
$$

and then $I(M, g) \geq e^{-C_{0}^{\prime}}(Y(M,[g]))^{2}$. This proves that there exists a positive constant $C=C(M, g)$ depending only on $(M, g)$ such that

$$
I(M, g) \geq C(Y(M,[g]))^{2} .
$$


Using this control from below of $I(M, g)$ we have that

$$
C \int_{M} e^{4 u_{t}} d V_{g} \geq \int_{M} \sigma_{2}\left(g^{-1} A_{g}^{1}\right) d V_{g}+\frac{1}{24}\left(\frac{7}{10}-t\right) C(Y(M,[g]))^{2}:=\mu_{t}>0 .
$$

This gives

$$
\max _{M} u_{t} \geq \log \mu_{t}-C(g) .
$$

Since $\left\|\nabla_{g} u_{t}\right\|_{g, \infty}<C_{1}$ this implies the Harnack inequality

$$
\max _{M} u_{t} \leq \min _{M} u_{t}+C(M, g),
$$

by simply integrating along a geodesic connecting points at which $u_{t}$ attains its maximum and minimum. Combining this two inequalities, we obtain

$$
\min _{M} u_{t} \geq \log \mu_{t}-C
$$

where $C$ only depends on $(M, g)$. This ends the proof of the Lemma.

\section{$4 C^{2, \alpha}$ estimate}

We have the following $C^{2, \alpha}$ estimate for solutions of the equation (1). For the proof, see [12] and [10].

Proposition 4.1 ( $C^{2, \alpha}$ estimate) Let $u_{t} \in C^{4}(M)$ be a solution of (2) for some $\delta \leq t \leq 2 / 3$, satisfying $\underline{\delta}<u_{t}<\bar{\delta}$, and $\left\|\nabla u_{t}\right\|_{g, \infty}<C_{1}$. Then for $0<\alpha<1,\left\|u_{t}\right\|_{g, C^{2, \alpha}} \leq C_{2}$, where $C_{2}$ depends only on $(M, g)$.

\section{Proof of the main Theorem}

We use the continuity method. Our 1-parameter family of equations, for $t \in\left[\delta, \frac{2}{3}\right]$, is

$$
\sigma_{2}^{1 / 2}\left(g^{-1} A_{u_{t}}^{t}\right)=f(x) e^{2 u_{t}}
$$

with $f(x)=\sigma_{2}^{1 / 2}\left(g^{-1} A_{g}^{\delta}\right)>0$, and $\delta$ was chosen so that $A_{g}^{\delta}$ is positive definite. Define

$$
\mathcal{S}=\left\{t \in\left[\delta, \frac{2}{3}\right] \mid \exists \text { a solution } u_{t} \in C^{2, \alpha}(M) \text { of (4) with } A_{u_{t}}^{t} \in \Gamma_{2}^{+}\right\} .
$$

Clearly, with our choice of $f, u \equiv 0$ is a solution for $t=\delta$. Since $A_{g}^{\delta}$ is positive definite, $\delta \in \mathcal{S}$, and $\mathcal{S} \neq \emptyset$. Let $t \in \mathcal{S}$, and $u_{t}$ be a solution. By Proposition 2.4, the linearized operator at $u_{t}, \mathcal{L}^{t}: C^{2, \alpha}(M) \rightarrow C^{\alpha}(M)$, is invertible. The implicit function theorem tells us that $\mathcal{S}$ is open. From classical elliptic theory, it follows that $u_{t} \in C^{\infty}(M)$, since $f \in C^{\infty}(M)$. By Proposition 2.5 we get an uniform upper bound on the solutions $u_{t}$, independent of $t$. We may then apply Proposition 2.6 to obtain a uniform gradient bound on $u_{t}$, and by Proposition 3.4, we get a uniform lower bound. Finally using Proposition 4.1 and the classical AscoliArzela's Theorem, then implies that $\mathcal{S}$ must be closed, therefore $\mathcal{S}=\left[\delta, \frac{2}{3}\right]$. The metric $\tilde{g}=e^{-2 u_{\frac{2}{3}}} g$ then satisfies $\sigma_{2}\left(A_{\tilde{g}}^{\frac{2}{3}}\right)>0$ and $R_{\tilde{g}}>0$. The positivity of the Ricci tensor associated to $\tilde{g}$ follows from proposition 3.2 (where $t$ is taken to be equal to $\frac{2}{3}$ ).

\section{Proof of Corollary 1.4}

Assume that $M$ admits a metric $g^{\prime}$ such that $Q_{g^{\prime}} \geq \frac{1}{48} R_{g^{\prime}}^{2}$ and $Y\left(M,\left[g^{\prime}\right]\right) \geq 0$. Recall that

$$
Q_{g^{\prime}}=-\frac{1}{4} \Delta_{g^{\prime}} R_{g^{\prime}}-2\left|R i c_{g^{\prime}}\right|_{g^{\prime}}^{2}+\frac{23}{32} R_{g^{\prime}}^{2}
$$

Integrating $Q_{g^{\prime}}$ on $M$ with respect to $d V_{g^{\prime}}$ we obtain (since $Q_{g^{\prime}} \geq 0$ )

$$
\int_{M}\left|R i g_{g^{\prime}}\right|_{g^{\prime}}^{2} d V_{g^{\prime}} \leq \frac{23}{64} \int_{M} R_{g^{\prime}}^{2} d V_{g^{\prime}}
$$


Now if we compute $\int_{M} \sigma_{2}\left(g^{\prime-1} A_{g^{\prime}}^{1}\right)$ using (5), we have (recall that $\left.\sigma_{2}\left(g^{\prime-1} A_{g^{\prime}}^{1}\right)=-\frac{1}{2}\left|R i c_{g^{\prime}}\right|_{g^{\prime}}^{2}+\frac{3}{16} R_{g^{\prime}}^{2}\right)$ :

$$
\int_{M} \sigma_{2}\left(g^{\prime-1} A_{g^{\prime}}^{1}\right) \geq \frac{1}{128} \int_{M} R_{g^{\prime}}^{2} d V_{g^{\prime}} \geq 0 .
$$

Now, consider the conformal laplacian operator $L_{g^{\prime}}:=\Delta_{g^{\prime}}-\frac{1}{8} R_{g^{\prime}}$. We have using the assumption $Q_{g^{\prime}} \geq \frac{1}{48} R_{g^{\prime}}^{2}$

$$
L_{g^{\prime}} R_{g^{\prime}}=\Delta_{g^{\prime}} R_{g^{\prime}}-\frac{1}{8} R_{g^{\prime}}^{2} \leq-8\left|R i c_{g^{\prime}}\right|_{g^{\prime}}^{2}+\frac{22}{8} R_{g^{\prime}}^{2}-\frac{1}{12} R_{g^{\prime}}^{2} \leq\left(-\frac{8}{3}+\frac{22}{8}-\frac{1}{12}\right) R_{g^{\prime}}^{2}=0 .
$$

Applying a Lemma due to Gursky [9], since $Y\left(M,\left[g^{\prime}\right]\right) \geq 0$ we have either $R_{g^{\prime}}>0$ (if $Y\left(M,\left[g^{\prime}\right]\right)>0$ ) or $R_{g^{\prime}} \equiv 0$ (if $Y\left(M,\left[g^{\prime}\right]\right)=0$ ). If $Y\left(M,\left[g^{\prime}\right]\right)>0$ we can apply our main Theorem to conclude that $M$ is diffeomorphic to a spherical space form. Otherwise, if $Y\left(M,\left[g^{\prime}\right]\right)=0$, since $Q_{g^{\prime}} \geq \frac{1}{48} R_{g^{\prime}}^{2}$ and $R_{g^{\prime}} \equiv 0$, we deduce, using the expression giving $Q_{g^{\prime}}$, that $R i c_{g^{\prime}} \equiv 0$ and then $M$ is diffeomorphic to a quotient of $\mathbb{R}^{3}$. This ends the proof of the Corollary.

\section{References}

[1] Besse, A.L. Einstein manifolds, Springer-Verlag, Berlin, 1987.

[2] Brendle S. and Schoen R., Manifolds with 1/4-pinched curvature are space forms. J. Amer. Math. Soc. 22 (2009), no. 1, 287-307

[3] Chang S.Y.A., Gursky M.J. and Yang P.C., An equation of Monge-Ampère type in conformal geometry, and four-manifolds of positive Ricci curvature, Ann. of Math. 155-3 (2002), 709-787.

[4] Chang S.Y.A., Gursky M.J., Yang P.C., A conformally invariant sphere theorem in four dimensions, Publ. Math. Inst. Hautes Études Sci. 98 (2003), 105-143.

[5] Djadli Z. and Malchiodi A., Existence of conformal metrics with constant $Q$-curvature. ArXiv : math.AP/0410141. To appear in Annals of Mathematics.

[6] Ge Y., Lin C.S. and Wang G., On the $\sigma_{2}$-scalar curvature. Preprint, 2007.

[7] Ge Y. and Wang G., On a fully nonlinear Yamabe problem. Ann. Sci. Ecole Norm. Sup. 39 (2006), 569-598.

[8] M.J. Gursky, Some local and non-local variational problems in Riemannian geometry, Séminaires et Congrès SMF, 4, 2000, 167-177.

[9] Gursky M.J., The Weyl functional, de Rham cohomology, and Kahler-Einstein metrics, Ann. of Math. 148 (1998), 315-337.

[10] Gursky M.J. and Viaclovsky J.A., Fully nonlinear equations on Riemannian manifolds with negative curvature, Indiana Univ. Math. J. 52 (2003), no. 2, 399-420.

[11] Gursky M.J. and Viaclovsky J.A., A new characterization of three-dimensional space forms, Inventiones Mathematicae, 145, 2001, 251-278.

[12] Gursky M.J. and Viaclovsky J.A., A fully nonlinear equation on four-manifolds with positive scalar curvature, J. Differential Geom. 63 (2003), no. 1, 2003, 131-154.

[13] Hamilton, Richard S., Three-manifolds with positive Ricci curvature, J. Differential Geom. 17 (1982), no. 2, 255-306.

[14] Paneitz S., A quartic conformally covariant differential operator for arbitrary pseudo-Riemannian manifolds, preprint, 1983.

[15] Rauch, H.E., A contribution to differerential geometry in the large, Annals of Mathematics, 54, $38-55$.

[16] Viaclovsky J.A., Conformal geometry, contact geometry, and the calculus of variations, Duke Kath. J. 101 (2000), no. 2, 283-316. 